\title{
La dinámica centro-periferia en el estudio de la ciencia en América Latina: notas para una reflexión historiográfica sobre la Argentina
}

\author{
Gabriel Matharan • \\ Centro Ciencia, Tecnología y Sociedad \\ de la Universidad de Maimónides, \\ Universidad Nacional del Litoral y \\ Universidad Autónoma de Entre Ríos
}

\section{Resumen}

El presente trabajo se inscribe en una investigación que indaga en las propuestas historiográficas desarrolladas, desde la década de 1980 hasta la actualidad, en el campo de Ciencia, Tecnología y Sociedad (CTS) en América Latina, para comprender la dinámica "centro-periferia» en el estudio de la ciencia en este continente. En particular aquí abordamos el estudio de las producciones académicas que son de referencia para la literatura CTS en la Argentina. Para ello, centramos el análisis en los conceptos elaborados para captar las relaciones entre el centro y la periferia. El trabajo muestra la riqueza de los planteos y su relevancia para los debates actuales sobre esta problemática.

\section{Palabras clave:}

Centro-periferia · Argentina · América Latina - historiografía

\begin{abstract}
This work is part of a research that explores the historiographical proposals developed in the field of Science, Technology and Society (STS) studies in Latin America from the 1980 s to the present. It aims to understand center-periphery dynamics in the study of science in Latin America. Here, we specifically examine reference academic outputs for STS literature in Argentina, focusing on the concepts developed to understand the relationships between the center and the periphery. The work shows the fertility of these proposals and their relevance to current debates on this issue.
\end{abstract}

\section{Key words:}

· Centre-periphery · Argentina · Latin America · historiography

\footnotetext{
- Licenciado en Historia (UNL), Magister en Ciencia, Tecnología y Sociedad (UNQ) y Doctor en Ciencias Sociales y Humanas (UNQ). Se desempeña como profesor en varias universidades. Pertenece al Centro Ciencia, Tecnología y Sociedad (Universidad Maimónides). Su interés de investigación gira en torno a la historia de la conformación de la química como disciplina en la Argentina y en América Latina. Además aborda problemas relacionados con la democratización del conocimiento científico. Actualmente se desempeña como director de la Red Latino-Americana de Investigación en Educación Química (ReLAPEQ).
} 


\section{Introducción}

En los últimos años, una cierta cantidad de literatura en los estudios de sociología e historia de la ciencia han puesto en cuestión, desde diferentes perspectivas, cierto uso rígido, estático y unidireccional del concepto centro-periferia que no permitió hacer visible el carácter diverso y situado (social e históricamente) y la dimensión nacional/local de la ciencia. ${ }^{1}$ Esto forjó una imagen distorsionada de la actividad científica en contextos considerados como periferias, como en el caso de América Latina.

En efecto, por mucho tiempo el desarrollo de la ciencia en esta región o bien no fue objeto de estudio o bien se la consideró como inexistente o se pensó que había recorrido, por imitación, las mismas etapas que el desarrollo científico de los países industrializados en Europa (Cueto, 1996:481). Entonces, las "periferias no europeas» fueron conceptualizadas como lugares sin tradición científica y de simple recepción del conocimiento científico (occidental), a la vez que Europa ocultaba que muchos de los presupuestos centrales de las prácticas culturales que reconocen como propias, fueron enunciados en la periferia (Said, 1978). Para dar cuenta de su implante se movilizó un modelo difusionista que centraba todo el peso de la explicación en las metrópolis o en centros de poder científicos europeos (Vessuri, 1996; Cueto, 1996; Saldaña, 2005).

La importancia de este modelo no fue solo cognitiva sino también política, en la medida que se tradujo en políticas científicas, y «guió el accionar de las agencias filantrópicas norteamericanas por muchos años, e inspiró a los primeros trabajos sobre la modernización científica que propusieron un modelo lineal de desarrollo de la ciencia» (Cueto, 1996:481). Ejemplo paradigmático fue el propuesto por George Basalla en 1967.

En este marco, el presente trabajo se inscribe en un programa de investigación que indaga en las propuestas historiográficas desarrolladas o adoptadas en la década de 1980, y en la actualidad, en el campo de Ciencia, Tecnología y Sociedad (CTS) en América Latina, y en campos de investigación relacionados, que dieron lugar a un entramado conceptual para iluminar el lugar de la dinámica "centro-periferia» a la hora de comprender y explicar cómo la ciencia, que es un producto europeooccidental, logró expandirse, localizarse y comenzar a practicarse en esta región en condiciones sociales y cognitivas específicas y diferentes respecto de los contextos institucionales más dinámicos en Europa occidental y Estados Unidos (Kreimer, 2000). En particular aquí se presenta un avance del estudio de obras de autores y autoras que han ejercido o ejercen una influencia en la literatura CTS producida en la Argentina, y que al mismo tiempo son relevantes a nivel latinoamericano en el establecimiento de la agenda de investigación sobre la problemática.

Con tal fin, en primer lugar, dada la importancia que tuvo durante mucho tiempo, e inclusive en la actualidad, tanto en la Argentina como en la región, se caracterizará el modelo de Basalla para dar cuenta del proceso de institucionalización de la ciencia en la periferia. En segundo lugar, se abordarán los primeros enfoques que, desde la periferia de América Latina, se plantearon para «descubrir» y reflexionar sobre la especificidad de la ciencia en esta región a partir de la tensión de los conceptos transmisión-apropiación y tradición-modernidad. En tercer lugar, se presentarán las perspectivas más recientes que centran su atención en la 
tensión integración-dependencia científica/académica mostrando las posibilidades y limitaciones de esta situación para la producción del conocimiento científico. Por último, en las conclusiones, se retomarán los aportes y las enseñanzas que se pueden extraer de las propuestas trabajadas a la vez que se reflexionará acerca de cómo los análisis centrados en la dinámica de circulación/tránsito y movilidad de la práctica científica de un lugar a otro, permiten enriquecer y complejizar el análisis de la «periferialidad» de la ciencia en la Argentina y en América Latina.

Para el análisis se tomaron los siguientes ejes: a) los conceptos elaborados; b) las relaciones entre el centro y la periferia que permiten captar estos conceptos; y, por último, c) las situaciones o casos concretos que a modo de base empírica ilustran a la vez que fundamentan las modalidades de estas relaciones. Vale la pena aclarar que si bien estos ejes refieren a un análisis en el plano de las ideas que expresa o contiene una obra, en un futuro trabajo se indagará en las condiciones sociales y políticas de surgimiento de las mismas y en el mutuo ensamblaje de lo social y lo cognitivo.

Por último, es importante señalar que hasta el momento no hay estudios que aborden, en términos historiográficos, cómo se reflexionó - y se reflexiona - a partir de la dinámica centro-periferia sobre el desarrollo de la ciencia en la Argentina. Se espera de esta manera realizar una primera contribución al conocimiento de esta problemática, que a la vez sirva de insumo para promover y desarrollar estudios comparados con otras regiones periféricas como, por ejemplo, la «europea periférica».2

\section{El modelo eurocéntrico y difusionista de Basalla}

A partir de la década del ' 60 se inició el interés por lo que podría denominarse el proceso de mundialización de la ciencia. Si bien es complejo analizar, como seńala Vallejos, el modo en que la comprensión socio-histórica de la localización de la ciencia en los países del tercer mundo, subdesarrollados o periféricos se vuelve objeto de estudio y de debate, debe notarse que los procesos de transformación de los «modos de existencia social de la ciencia» (Pestre, 2005) suelen ir acompañados también por transformaciones en los temas que aparecen relevantes para la comprensión de la propia ciencia. En la medida en que la ciencia tomaba forma internacional a partir de las políticas de cooperación científica y técnica impulsadas por algunos organismos multilaterales como la UNESCO, se abrió también un interés por los procesos de internacionalización de la ciencia (Vallejos, 2010).

Sin simplificar la multiplicidad de los "puntos de emergencia», se reconoce que uno de los primeros intentos conocidos por explicar la mundialización o el proceso de expansión de la ciencia europea fue emprendido por Basalla con su texto The Spread of Western Science de 1967 (Lafuente et al., 1993). Este autor propone una teoría del cambio, de cómo se pasa de un momento pre científico a uno científico. En efecto, propuso un modelo interpretativo concebido en tres fases (de contacto, ciencia colonial y ciencia independiente) que se basa en la noción de «difusión o mundialización» de la ciencia occidental desde un polo - Europa - hacia las sociedades no científicas que poco a poco van construyendo este tipo de cultura. 
En la primera fase, la sociedad o nación no científica se constituye como fuente de información para las naciones que ya disponen de ciencia. La visita de científicos europeos permite la exploración de nuevas tierras, de su flora y fauna. Este movimiento culmina a inicios del siglo XIX con los trabajos de Alexander von Humbolt y Charles Darwin. Durante la segunda fase emerge la denominada "ciencia colonial», entendida como ciencia dependiente. Ahora el científico colonial puede ser un criollo o un europeo emigrado. Es dependiente en la medida que la cultura científica de este científico criollo no es local sino proveniente del exterior y que está en una situación de absoluta dependencia cultural en términos de temas de investigación, lugares para publicar, instituciones para formarse, signos y credenciales de reconocimientos y de consagración, etc. Esta ciencia colonial no es una ciencia inferior y no supone la existencia de una relación colonial formal. En la última fase se conforma una tradición científica (cultural) independiente. Ahora el científico se centra en su propio medio científico, en el cual recibe la formación, reconocimiento, remuneración, establece sus comunicaciones y formula sus líneas de trabajo. Permanece en contacto con el exterior, pero ahora el peso específico de éste es otro. Para llegar hasta allí han de vencerse obstáculos de orden religioso y filosófico inherentes a la cultura nacional, recibir apoyo del estado, desplegar una enseñanza científica, promover instituciones, facilitar las comunicaciones y crear una base tecnológica propia (Basalla, 1967).

El tránsito de la fase dos a la tres es explicada por Basalla por elementos contextuales, como el sentimiento nacionalista de los científicos que se combina con componentes tanto culturales como políticos de su medio de pertenencia. Sin embargo, lo esencial, a su juicio, es un factor interno a la propia ciencia: la lucha por una cultura científica propia.

Este modelo es caracterizado como «eurocéntrico» $\mathrm{y}$ «difusionista» ya que sólo reconoce a las periferias no europeas sin tradición científica como laboratorios europeos para hacer ciencia y como lugares de simple recepción del conocimiento científico occidental desplazando a las formas locales de conocimiento ${ }^{3}$. Con esto no sólo se despreció el conocimiento que las culturas no europeas habían formado antes de la implantación de la ciencia, sino que, inclusive, se ignora la legitimidad y significación de este saber hoy (Vessuri, 2014).

Propone, además, que la difusión es el resultado de la inexorable unidireccionalidad de la diseminación de ideas elaboradas en un «centro» geográficamente localizado hacia una periferia de contornos difusos, con ocupantes a menudo anónimos y, en general, escasamente conocidos, cuya única actitud posible fue la de aceptar o rechazar las nuevas ideas. Así, el emisor (que difunde, propaga, esparce, divulga y extiende) aparece como la parte activa y el receptor tiene un carácter pasivo. Esta imagen se apoya en una concepción de los procesos de transmisión de la ciencia en los que la aceptación de nuevas ideas se entiende como un hecho natural, fruto del reconocimiento objetivo de la verdad por parte de personas de mente abierta. Abona, además, a una idea de ciencia universal, neutral y un modelo explicativo de carácter internalista. Desde este punto de vista, sólo la crítica y el rechazo merecen ser explicados, tratando de identificar los prejuicios o los oscuros intereses que condujeron a tales resistencias al progreso científico.

A nivel internacional el trabajo de Basalla sufrió críticas y «traducciones» a fines de la década de 1980 (Reingold y Rothenberg, 1987; Mcleod, 1987; Polanco, 1990; Chambers, 1993; Lafuente y Catalá, 1989). En 1993 tuvo lugar en Madrid 
(España) un congreso internacional denominado Mundialización de la ciencia y cultura nacional, destinado a discutir la tensión internacional-nacional presente en la ciencia. Aquí Basalla presentó un revisión de trabajo, The Spread of Western Science revisited, en donde discutió las interpretaciones que recibió su propuesta de 1967 (Lafuente et al. 1993).

Lo que resulta relevante del texto de Basalla es que su artículo coloca como cuestión central la doble existencia de la ciencia: es una empresa a la vez internacional y local. Ello dio lugar a la paulatina formación de una literatura sobre la ciencia colonial y la ciencia nacional en regiones periféricas (Mcleod, 1987; Chambers, 1993; Lafuente y Catalá, 1989).

\section{2. ¿Ciencia periférica o ciencia en la periferia?}

En la década de 1980, en Europa y Estados Unidos, tuvo lugar la emergencia de las nuevas corrientes que genéricamente podemos llamar constructivistas (Shinn, 1999). Estas removieron, en primer lugar, la creencia de que la ciencia constituye una esfera autónoma de operaciones intelectuales, disolviendo la distinción tradicional entre los contextos de justificación y del descubrimiento. En segundo lugar, criticaron el carácter universalista de la ciencia, enfatizando su carácter contextual. Por último, establecieron dudas sobre el pretendido carácter privilegiado y fundamentado del conocimiento científico para acceder al mundo natural y social. Para ello establecieron algunas hipótesis entre el contexto social de emergencia de las prácticas científicas, las relaciones internas en el ámbito de producción de conocimientos y los contenidos de los mismos. Esto permitió hacer visible que la producción y la movilidad del conocimiento son procesos simultáneos.

En América Latina, la teoría del desarrollo, que sostenía la noción de que la ciencia y las universidades jugarían un papel importante en el desarrollo socioeconómico de la región, y la teoría de la dependencia, que reflexionó sobre el carácter periférico y dependiente, en términos económicos, de los países latinoamericanos, constituyeron un telón de fondo sobre el cual comenzó a reflexionarse sobre la ciencia. Entonces, el interés por capturar el modo en que la ciencia se localizó en la región fue acompañado de un interés político tanto por posicionar a América Latina en el concierto mundial de la ciencia como por gobernar su destino.

La primera vez que apareció la denominación ciencia periférica fue en un libro escrito en Venezuela en 1983, La Ciencia Periférica, dirigido a comprender la relación que la ciencia mantenía con la sociedad en este país. En la introducción, la historiadora argentina-venezolana Hebe Vessuri, haciéndose eco de los nuevos planteos constructivistas, abogaba por la necesidad de reconocer las especificidades de los contextos nacionales a la hora de explicar la dinámica de la ciencia. Así, luego de abordar la influencia del contexto sociocultural sobre la misma, concluyó que la ciencia en Venezuela y en América Latina era periférica debido a tres razones.

La primera razón es que el desarrollo conceptual tiene menos posibilidad de ocurrir en América Latina por los riesgos que supone la creación de conocimiento 
verdaderamente nuevo, tanto en términos de costo económico como intelectual. Las comunidades científicas de la periferia son más conservadoras que en los centros, trabajan casi exclusivamente dentro de los parámetros de la ciencia «normal», en la resolución de rompecabezas cuya concepción fundamental se da en otras partes. La segunda razón, referida al nivel de los temas de investigación, radicaba que el aporte que están en condiciones de hacer los científicos de la periferia, especialmente en disciplinas «maduras», está más en la «aplicación» de una ciencia, orientada por necesidades sociales, que en una verdadera "ciencia pura" percibida como "más científica». El caso más significativo sería aquí el de la medicina. La última razón se ubica en el nivel de las instituciones científicas, lugar que determina los métodos de trabajo, los modos de transferencia y difusión de la información. Ella sería la expresión concreta de la interacción de modelos internacionales con los intereses ideales y mentalidades diferentes de los actores científicos locales. Como resultado de esta interacción, las instituciones construyen su propia política que da forma al modo de producción de los conocimientos científicos.

La Ciencia Periférica dio lugar a otras obras en donde se abordó la constitución de las disciplinas científicas en el medio académico (1984) y las características que asumieron las instituciones científicas en la historia de la ciencia en Venezuela $(1987)^{4}$. De esta forma, en 1984, Vessuri publicó la Ciencia Académica en la Venezuela Moderna, obra en la que se puede apreciar una flexibilización de la postura presentada en 1983, donde los contextos socioculturales ubicados en la periferia parecía que operaban como una restricción fundamental para el aporte conceptual y el establecimiento de los temas de investigación (Kreimer, 2000).

El supuesto básico que se presentaba y se defendía ahora era la idea de que el conocimiento científico cruzaba límites internacionales, es decir se movía de un lugar a otro, dando lugar a un intercambio. Lo que interesaba ver particularmente eran los motivos de esta movilidad, las mecanismos que se usaban para mover el conocimiento, qué ocurría en la medida que cruzaba esos límites, y la efectividad de su uso. Esta idea se mantendrá en escritos posteriores y será una idea central de toda su producción intelectual. Como señaló varios años más tarde, «la ciencia occidental dio lugar a un conjunto muy poderoso de dispositivos sociales para validar el conocimiento y, de hecho, para mover el conocimiento de unos sitios a otros» (Vessuri, 1996:221). En consecuencia, si se reconocía que el nacimiento de lo que llamamos ciencia occidental fue inseparable de su expansión/movilidad, el lugar de las periferias adquiriría un significado fundamental, muy diferente del lugar marginal y accesorio que el que venían ocupando. Para poder considerar esta especificidad, era necesario entonces:

revisar las condiciones de transferencia de modelos epistemológicos y contenidos disciplinarios y los medios utilizados para transferirlos, al igual que la recepción, interpretación y utilización de esos conocimientos en la sociedad receptora, tomando en cuenta la naturaleza interactiva del intercambio de conocimiento y por ende la participación local, en la reproducción y desarrollo de disciplinas y especialidades particulares. (33)

El enfoque partía de considerar que: a) la ciencia es una poderosa institución internacional, que depende básicamente para su éxito de insumos de los centros del sistema mundial; b) la ciencia es un objeto de transferencia de los países desarrollados a los periféricos, a los efectos de incorporar a estos últimos como apéndices 
culturales, además de económicos y políticos; c) existen entre los países centrales y los periféricos asimetrías de poder, y en consecuencia desbalances en los intercambios de conocimientos, que impliquen que las denominadas modernizaciones del conocimiento sean en realidad formas de borrar el saber local previo como posible conocimiento alternativo que pudiera construirse; $y, d$ ) aún cuando se empiece a participar de la ciencia como una cultura dominante, se ofrecen resistencias apropiando-transformándola, con objetivos generalmente nacionalistas, a partir de la asociación ideológica entre ciencia y desarrollo (Vessuri, 1984).

Doce años más tarde, en 1996, Vessuri publicó un artículo llamado «La institucionalización científica», en donde citó por primera vez el texto de Basalla. Si bien adoptó sus conceptos de ciencia colonial y ciencia nacional y periodización, ofreció un planteo alternativo. En efecto, propuso pensar el proceso de ingreso y desarrollo de la ciencia en los países periféricos no en términos de transmisión sino de apropiación. Cuestionó el modelo que sostenía que la ciencia se transmitía unilateralmente del centro hacia la periferia y que en esta transmisión la periferia fuera pasiva a la hora de «recibir» las nuevas ideas y prácticas. Desde este punto de vista, el proceso de institucionalización científica fue entendido como el

proceso mediante el cual han surgido las tradiciones científicas nacionales modernas dentro de los contextos sociales más variados en las naciones poscoloniales, donde las instituciones científicas han representado en distintas épocas las múltiples manifestaciones de patrones específicos de respuestas culturales y económicas ante la compleja combinación de ideas y sucesos que se identifican como la ciencia occidental. (200)

Para hacer visible este complejo proceso, centró su indagación en las estrategias y estilos de las grandes potencias a la hora de exportar la ciencia a sus colonias, la riqueza de respuestas culturales dadas por éstas, mediante las cuales la ciencia occidental se asimilaba o se rechazaba, las disciplinas e instituciones de la ciencia colonial, las condiciones para el surgimiento de la ciencia nacional y la definición contextual de sus normas y formas de organización. En consecuencia, para esta autora, a la hora de explicar la constitución de tradiciones científicas (durante la época colonial y la nacional) es importante considerar no sólo los intereses académicos, administrativos, comerciales y militares de las metrópolis para exportar la ciencia occidental a sus posesiones coloniales, y con ello aumentar su dominio, sino también los esfuerzos de las élites locales por dominar los conocimientos que prometían modernidad.

Por su parte, el historiador peruano Marcos Cueto presentó su investigación sobre la trayectoria de investigadores e instituciones pertenecientes a la fisiología andina en el Perú en su obra Excelencia científica en la periferia. Actividades científicas e investigación biomédica en el Perú, 1890-1950.5 A partir de este caso, y dialogando con el trabajo citado de Vessuri de 1983, prefiere señalar la distinción entre la ciencia periférica y lo que él denomina ciencia «en» la periferia y sobre todo, de «excelencia científica en la periferia». Con ello buscaba resaltar que

no toda la ciencia de los países atrasados es marginal al acervo mundial del conocimiento y que el trabajo científico tiene en estos países sus propias reglas que deben ser entendidas no como síntoma de atraso o modernidad, sino como parte de su propia cultura y de las interacciones con la ciencia internacional. (Cueto, 1989:28) 
La cuestión que se formula aquí remite a una tensión: cómo comprender la combinación de un trabajo moderno y creativo en un contexto cultural supuestamente tradicional y "periférico» a los centros mundiales de la ciencia. Además, introduce una importante contextualización histórica al sostener que es necesario recordar que la distancia que hoy existe entre la ciencia de los países desarrollados y la de algunos países subdesarrollados no fue tan amplia en el pasado, y que más bien esta separación ha tendido a crecer en los últimos cuarenta años. En contexto, sostuvo que "la ciencia periférica no tiene porqué seguir siendo periférica» (Cueto, 1989: 190). Esto plantea la cuestión de por qué esta distancia se fue haciendo cada vez más amplia y si los casos señalados por Cueto como de excelencia científica pudieron haber sido excepciones.

¿Qué aportaría el concepto de ciencia en la periferia? Según Kreimer,

pone de manifiesto el carácter heterogéneo de las comunidades científicas locales que el concepto de ciencia periférica tiende a desdibujar, y no sólo parece borrar las diferencias «en el interior» de comunidades científicas particulares, sino también entre varias comunidades científicas localizadas en contextos claramente diferenciados. $(2000,2010)^{6}$

En un trabajo posterior, Cueto también hizo referencia de manera crítica al trabajo de Basalla pero, a diferencia de Vessuri, no usó sus conceptos ni sus periodizaciones. En cambio, ahondó en los elementos contextuales y las estrategias desarrolladas por los investigadores locales para alcanzar un reconocimiento internacional trabajando en su país periférico. Distingue cinco tipo de estrategias: 1) concentración de recursos institucionales y humanos; 2) la combinación de investigación básica y aplicada; 3) el nacionalismo, que pudo haber afectado la selección de los tópicos y eventualmente el contenido de la ciencia; 4) la búsqueda de clientes, especialmente locales; 5) la utilización de una tecnología de investigación no sofisticada; y 6) una temprana inserción en el contexto internacional (Cueto, 1997).

\section{Perspectivas recientes: ¿integrados o dependientes?}

En la primera década del siglo XXI, tanto en América Latina como en Europa, se empezaron a revisar y problematizar las nociones de ciencia periférica por su carácter estático, ahistórico y por su capacidad explicativa limitada e insuficiente (Fox, 2003; Gavroglu et al., 2008; Kreimer, 2010). En efecto, la «condición periférica» de los países subdesarrollados aparece como un punto de partida, como un hecho inexorable, determinado por una causalidad externa. Estos trabajos no trataron de negar la existencia de «centros» sino de cuestionar su absolutización. Es decir, dejar de utilizar estas nociones de "centro" y "periferia» como factores explicativos, para adoptar una perspectiva que las considere como resultado de una dinámica relacional construida y reproducida social y cognitivamente. Como señalan Dagnino y Thomas, lejos de ser una causa exogenerada, la condición periférica es un efecto que responde a causalidades 
endógenas de la dinámica local (Dagnino y Thomas, 2000). Así, se pueden encontrar centros y periferias que, dependiendo de los temas de investigación, pueden ser a la vez centros y periferias; centros que puede convertirse, con el tiempo, en periferias y viceversa, y puede haber países que sean centros y periferias a la vez (Gavroglu, et al., 2008).

Dentro de estos planteos, e inscribiéndose en la tradición de investigación de la dinámica centro-periferia en América Latina, encontramos la posición de Pablo Kreimer, quien propuso abordar este problema a partir de la conformación de «tradiciones científicas» en la periferia (Kreimer, 2000, 2009). Afirma que si las observamos veremos que, en la mayoría de los casos, se construyen con vínculos más o menos fuertes respecto de tradiciones localizadas en países centrales (Kreimer, 2010). Por lo tanto, el análisis de estas relaciones no es un elemento más, sino que resulta crucial para comprender la dinámica de la ciencia en las periferias.

Al historizar estas relaciones en el marco de lo que denomina «universalización liberal», ${ }^{7}$ período que va desde comienzos del siglo XX hasta la década de 1980 (Kreimer, 2013), se evidencia que su mecanismo típico de conformación se da a través de una "integración subordinada». La expresión tiene dos partes: «la primera opondría "integración" a "marginalidad" o "aislamiento". La segunda, opondría "subordinada" a "relación entre pares" o "independencia" (Kreimer, 2009:131). ${ }^{8}$ Este concepto reconoce la existencia de asimetrías de poder en el campo de la ciencia que se hace visible en una división social del trabajo científico y el desarrollo de una «ciencia hypernormal que indaga hasta los más mínimos detalles de un fenómeno en particular, sin poder realizar ningún aporte significativo en términos conceptuales o teóricos» (Kreimer, 2000).

Esta dinámica es característica del modo en que surgen nuevas áreas de indagación en contextos periféricos, generándose muchas veces la replicación de las agendas de investigación internacional a partir de la experiencia del investigador local formado en el exterior. Al mismo tiempo, éste opera como un proveedor de datos para el laboratorio central que lo acogió. Así, el grupo central ejerce el control cognitivo del tema en cuestión y el control de las aplicaciones posibles de los conocimientos producidos (Kreimer, 2000, 2013). En efecto, la integración supone la asignación de un trabajo a actividades de alto contenido y especialización técnica, pero que son subsidiarias de problemas científicos y/o productos ya definidos previamente. Si bien son actividades importantes para el desarrollo global del problema, no implican per se avances significativos en términos conceptuales. Mas esta integración es subordinada, en la medida en que se tiene un escaso margen de negociación para la definición y elección de las líneas de investigación, la visión de conjunto de los problemas conceptuales y también sus utilidades reales o potenciales. De esta manera, las periferias están sometidas a una fuerte dependencia de los dictados de los centros de referencia localizados en los países más desarrollados.

En la actualidad, sin embargo, ha surgido una nueva dinámica de producción del conocimiento científico en el marco de la constitución de megarredes concentradas conformadas a partir de las últimas décadas del siglo XX. Las mismas dan lugar a una integración subordinada, con un creciente aumento de peso de las fuerzas que producen la subordinación, disminuyendo cada vez más los márgenes de negociación y de resistencia (Kreimer, 2013). Esto obedece a modificaciones de las políticas de Ciencia y Tecnología de los países desarrollados, cambios en las 
modalidades de las colaboraciones entre los investigadores a partir de los medios electrónicos y cambios en la naturaleza de la escala de la investigación misma.

En contrapartida, la nueva modalidad de integración subordinada permite, en primer lugar, que los grupos de investigación locales cuenten con un alto grado de información y con ello puedan participar en las discusiones sobre el estado de las investigaciones sobre la temática abordada. En segundo lugar, que puedan acceder a financiamientos de origen internacional, a los cuales de otro modo difícilmente podrían. Por último, posibilita que adquieran una mayor legitimidad local diferenciándose de aquellos grupos no integrados internacionalmente que, en consecuencia, se encuentran aislados o, en el mejor de los casos, reproducen las relaciones de integración subordinada, pero esta vez en el interior del país (Kreimer, 2013).

Nutriéndose y aportando a los estudios CTS, los trabajos que tienen como objeto de indagación la "dependencia académica» también han problematizado y han aportado reflexiones sobre las comunidades científicas periféricas. Como afirman Beigel y Sabea, por dependencia académica se entiende «la estructura desigual de producción y difusión del conocimiento construida históricamente en lo que conocemos como sistema científico internacional» (Beigel y Sabea, 2014:15). Desde una perspectiva periférica lo que buscan mostrar estos trabajos es hasta qué punto la estructura desigual de ese sistema incidió (y sigue incidiendo) en la producción de conocimiento de las comunidades científicas periféricas, en la constitución de capacidades para construir conceptos innovadores y definir una agenda de investigación propia y en sus posibilidades de hacer circular internacionalmente sus conocimientos (Beigel, 2010). En este contexto se habla de «dependencia académica» para referirse a situaciones de dominación que devienen de la posición de un campo académico en el sistema académico mundial.

Lo que resulta relevante de esta conceptualización, producida desde la perspectiva periférica, es que no se puede hablar sin más de dominación ya que esto anularía toda la capacidad de autodeterminación intelectual a los países dominados. Si fuera así, negaría la posibilidad de hablar de la existencia misma de «campos» fuera de las academias centrales.

Entonces no se puede interpretar la dependencia académica simplemente como un reflejo o vínculo vertical que une a productores activos con reproductores pasivos (Beigel y Sabea, 2014). El hecho de que el conocimiento producido en las comunidades periféricas cuente con tasas bajas de circulación dentro de los sistemas académicos internacionales no implica que su producción sea —o haya sido siempre - el resultado de la importación irrestricta de conceptos, recursos extranjeros o la división internacional del trabajo. Las ciencias sociales latinoamericanas desarrollaron estrategias para superar la dependencia académica alcanzando en la década de 1960 un alto nivel de desarrollo institucional y autonomía intelectual. Se generó un vigoroso circuito subregional y se consolidaron sistemas universitarios públicos de gran tamaño en Brasil, México, Argentina y Chile, surgiendo tradiciones académicas locales que adquirieron cada vez más fuerza. Esto no implicó, por supuesto, que los conocimientos producidos superasen las jerarquías impuestas por el sistema académico mundial, ni alcanzaran altos niveles de circulación internacional o de exportación hacia los centros (Beigel, 2010). Se muestra que hasta 1973 se constituyó y funcionó un circuito sudamericano de las ciencias sociales que alcanzó una posición dominante a nivel regional, aunque mantuvo una posición dominada en el sistema académico mundial. Aquí tuvo lugar la construcción de 
un paradigma científico enraizado, la teoría de la dependencia, que se montó en base a tradiciones preexistentes en la región y dialogó críticamente con las ciencias sociales occidentales. Todo lo cual permite suponer que «no hay "exportador" puro de teorías y un "importador" pasivo de las mismas; los intercambios simbólicos, por más desiguales que sean, no son unilaterales» (Beigel, 2010:22). Y que

la autonomía académica de los circuitos periféricos no puede ser medida en una perspectiva internacional comparativa en función de supuestos niveles de «originalidad científica» (y mucho menos en función de la participación en los medios de consagración concentrados por las academias centrales), sino en relación con la capacidad endógena de estas comunidades de producir conocimientos y participar en formas alternativas de circulación. (18)

\section{A modo de cierre: la persistencia del problema}

Del análisis de los autores que se han trabajado y que critican el modelo de Basalla, se puede apreciar que ya en los años 80 Vessuri planteaba la necesidad de considerar la circulación del conocimiento científico y el concepto de apropiación para entender la dinámica centro-periferia. En este contexto el concepto de periferia, de carácter cognitivo a la vez que político, sigue siendo útil para ubicar/describir comunidades científicas que estaban consideradas (y actualmente lo están) en las afueras o distantes de los centros del conocimiento, a condición de que pueda ponderar la relación entre lo local y lo internacional en la ciencia y se reconozca que «el carácter internacional de la ciencia estaría producido por una compleja, inacabable interacción de diversos estilos nacionales o regionales. La ciencia (universal) no sería más que el conjunto de ciencias nacionales» (Vessuri 1993:732). Además, según Vessuri, los usos del concepto de periferia que se han realizado hasta el momento tienen más bien un carácter descriptivo para ubicar diferentes grupos, instituciones o la ciencia de un país en un mismo sistema intelectual, universitario o científico. Esta situación abre una cuestión específica: «¿Cómo hacer pasar el término periférico del nivel existencial al nivel teórico?» (727).

Por otra parte, los intentos de escapar a las visiones eurocéntricas y norteamericanocéntricas de la historia de la ciencia y la necesidad de hacer posibles y perceptibles otras historias no pueden reducirse a negar la importancia de Europa y de los Estados Unidos en la historia moderna. Si bien en el presente no hay un centro sino una variedad de centros de distinto peso e influencia, con vectores que apuntan en distintas direcciones, aun así, hay centros de centros y Estados Unidos sigue conteniendo una gran cantidad de centros institucionales y disciplinarios (Vessuri, 2007).

Dicho esto es necesario resaltar que las relaciones centro-periferia no sólo son cambiantes en el tiempo y en función de disciplinas, sino que también las periferias no son pasivas en su relación con el conocimiento científico. Su rol activo queda al menos plasmado en los procesos de integración que, más allá de la subordinación o dependencia (académica) respecto de los centros, implican procesos de apropia- 
ción y de resistencia frente a la imposición, explícita o implícita, de modalidades de prácticas científicas. Esto también abre una agenda de investigación con un mayor énfasis en el estudio de casos ligados a las modernas periferias, una mayor insistencia en los procesos de circulación, intercambio y localización de teorías, prácticas de enseñanzas, equipos, bibliotecas, profesores, etc. (Raj, 2006; Vessuri, 2007; Podgorni 2007), en las modalidades de apropiación y resistencia y en la identificación de las condiciones cognitivas y políticas de los posibles intercambios (desiguales) de los mismos — con los que se insiste en la multidireccionalidad de los mismos frente a la unidireccionalidad de los procesos de difusión, transmisión. ${ }^{9}$ Esta última condición no invalida que la consideración del desarrollo de la ciencia en los contextos no europeos, como la Argentina, y de manera más general en América Latina, no pueda enriquecer los análisis sobre este proceso a nivel internacional, a la vez que pensar en qué medida esos desarrollos contribuyen en la actualidad, al igual que en el siglo XIX, también a lo que sucede en los países centrales o cómo han surgido «centros periféricos» que tuvieron un rol destacado en la región.

A modo de síntesis, si reconocemos que el nacimiento y constitución de lo que llamamos ciencia occidental es inseparable de su expansión/movilidad hacia todas las regiones del planeta, entonces hace falta resaltar más intensamente el lugar de las periferias en este proceso. En este contexto, una visión más compleja de la dinámica actual de la ciencia requeriría hacer visible, en la medida en que así fuera, cómo desde las periferias se construyen los centros (Belmar y Bertomeu Sánchez, 2003), mostrando el carácter constitutivamente híbrido de éstos (Chakrabarty, 1989). Hacer visible esto, quizás, sea el gran desafío para los futuros estudios sobre la ciencia en la Argentina y en la región. 
tarea "exitosa" o "moderna" y por ello menos periférica, puede resultar en una interpretación sesgada» (2000).

7 «Por liberal se entiende aquí el ejercicio de prácticas que no son reguladas por las autoridades nacionales ni por la dirección de instituciones. Se trata, en cambio, de prácticas marcadas por un laissez-faire que no corresponde más que a las estrategias de los propios investigadores» (Kreimer, 2013:437).

${ }^{8}$ Las características de estas tradiciones científicas, prácticas modernas a la vez que periféricas, permiten problematizar la naturalización de las oposiciones como moderno-central y arcaico-periférico. En este sentido, es en la no correspondencia donde se encuentran la riqueza y los matices (Kreimer, 2010).

${ }^{9}$ Resultan de especial interés, en el denominado "giro geográfico" para el estudio de la ciencia, los trabajos de Livingstone (2003), que indaga en las nociones de «sitio», «región», «circulación» y de Salvatore (2007) en donde se problematizan los «lugares» del saber. También el trabajo de Secord (2004) que entiende la ciencia como una forma de acción comunicativa (communicative action).

\section{Referencias bibliográficas}

Basalla, G. (1967). The Spread of Western Science. Science, (156), 611-622.

(1993). The Spread of Western Science revisited. En Lafuente, A. et al., Mundialización de la ciencia y cultura nacional (pp. 559-603). Madrid: Doce Calles

Belmar García, A. y Bertomeu Sánchez, J.R. (2003). Constructing the Center from the Periphery. Spanish Travellers to France al the Time of the Chemical Revolution. En Simoes, A. et al. (Eds.), Travels of Learning. A Geography of Science in Europe. Dordrecht: Kluwer.

Beigel, F. (Dir.) (2010). Autonomía y dependencia académica. Universidad e investigación científica en un circuito periférico: Chile y Argentina (1950-1980). Buenos Aries: Biblos.

Beigel, F. y SABEA, H. (2014). Dependencia académica y profesionalización en el Sur. Perspectivas desde la periferia. Mendoza: Ediunc.

Chambers, D. (1987). Period and Process in Colonial and National Science. En Reingold, N. y Rothengberg, M. (Eds.), Scientific Colonialism: A Cross Cultural Comparison (pp. 297-321). Washington, D.C.: Smithsonian Institution Press.

(1993). Locality and science: myths of centre and periphery. En Lafuente, A. et al., Mundialización de la ciencia y cultura nacional (pp. 605-618). Madrid: Doce Calles.

Chakrabarty, D. (1989). Rethinking Working-Class History. Bengala 1890-1940. Princeton y Oxford: Princeton University Press.

CuEto, M. (1989). Excelencia cientifica en la periferia. Actividades científicas e investigación biomédica en el Perú, 1890-1950. Lima: GRADE. (1996). La excelencia en las ciencias biomédicas del siglo XX. 
En Saldaña, J. J. (Comp.), Historia social de las ciencias en América Latina (pp. 481-492). México: UNAM/Miguel Ángel Porrúa.

Dagnino, R. y Thomas, H. (2000). Elementos para una renovación explicativa-normativa de las políticas de innovación latinoamericanas. Espacios, 21 (2).

DíAs, E. ET AL. (1983). La ciencia periférica. Caracas: Monte Ávila Editores. Fox, R. (ED.) (2003). Centre and Periphery Revisited: The Structures of European Science, 1750-1914. Revue de la Maison française: Oxford. Gavroglu, K. ET AL. (2008). Science and Technology in the European Periphery: Some Historiographical Reflections. History of Science, 46, 153-175. Kreimer, P. (2013). Internacionalización y tensiones para un uso social de la ciencia latinoamericana. Del siglo XIX al siglo XX. En Restrepo Forero, O. Ensamblando estados (pp. 437-452). Bogotá: Universidad Nacional de Colombia, Facultad de Ciencias Humanas, Centro de Estudios Sociales (CES).

- (2010). Nacimiento, muerte y resurrección de la biología molecular en la Argentina. Aspectos sociales, politicos y cognitivos. Buenos Aires: Eudeba. - (2000). Ciencia y periferia. Una lectura sociológica. En Montserrat, M. (Comp.) La ciencia argentina entre siglos (pp. 187-202). Buenos Aires: Manantial.

(2009) El cientifico también es un ser humano. La ciencia bajo la lupa. Buenos Aires: Siglo XXI.

Lafuente, A. y Sala Catalá, J. (1989). Ciencia colonial y roles profesionales en la América Española del s. XVIII. Quipu, 6(3), 387-395. Lafuente, A. y Ortega, M.L. (1992). Modelos de Mundialización de la ciencia. Arbor, CXLII (558-559-560) 93-117.

LAFUENTE, A. ET AL. (EDs.) (1993). Mundialización de la ciencia y cultura nacional. Madrid: Doce Calles.

Macleod, R. (1987). On Visiting the "Moving Metropolis»: Reflections on the Architecture of Imperial Science. En Reingold, N. y Rothengberg, M. (Eds.), Scientific Colonialism: A Cross Cultural Comparison (pp. 217-249). Washington, DC: Smithsonian Institution Press.

(1993) The worldwide diffusion of science. En Lafuente, A. et al. (Eds.), Mundialización de la ciencia y cultura nacional (pp. 735-738). Madrid: Doce Calles.

Pestre, D. (2005). Ciencia, dinero y politica. Buenos Aires: Nueva Visión. (Traducción al español de R. Figueira: Science, argent et politique. Un essai d'interprétation, París: INRA, 2003.)

Podgorny, I. (2007). De ángeles, gigantes y megaterios. El intercambio de fósiles de las provincias del Plata en la primera mitad del siglo XIX. En Salvatore, R. (Comp.) Los lugares del saber. Contextos locales y redes transnacionales en la formación del conocimiento moderno (pp. 125-152). Rosario: Beatriz Viterbo Editora.

Polanco, X. (ED.) (1990). Nasissance et développment de la sciencie-monde: Production et reproduction des communautés scientifiques en Europe et Amérique Latine. Paris: La Découverte

Pyenson, L. (1985). Culture Imperialism and Exact Sciencies: German Science Expansion Overseas, 1900-1930. Nueva York: Peter Lang. 
(1993). Civilizing Mission. Exact Sciences and French Overseas Expansion, 1830-1940. Baltmore y Londres: The John Hopkins University Press.

RaJ, KapiL (2006). Relocating Modern Science: Circulation and the Construction of Scientific Knowledge in South Asia and Europe: Seventeenth to Nineteenth Centuries. Delhi: Permanent Black.

Reingold, N. \& Rothengberg, M. (Eds.). Scientific Colonialism: A Cross Cultural Comparison. Washington, DC: Smithsonian Institution Press. SAID, E. (1978). L'orientalisme. L'Orient créé par L'Occident. Paris: Seuil. Saldaña, J.J. (2005). La Casa de Salomón en México. Estudios sobre la institucionalización de la docencia y la investigación cientifica. México: Facultad de Filosofía y Letras, UNAM.

Salvatore, R. (2007). Los lugares del saber. Contextos locales y redes transnacionales en la formación del conocimiento moderno. Rosario: Beatriz Viterbo Editora.

SECORD, J. (2004). Knowledge in Transit. Isis, (95), 654-672.

VAlLejos, O. (2010). La ciencia en el litoral: las modalidades de localización de la ciencia en Santa Fe. Revista de la Junta Provincial de Estudios Históricos de Santa Fe, (LXVIII), 173-198.

Vessuri, H. (Cомr.) (1984). Ciencia Académica en la Venezuela Moderna. Caracas: Fondo Editorial.

(1987). Las instituciones cientificas en la historia de la ciencia en Venezuela. Caracas: Fondo Editorial Acta Científica Venezolana.

(1993). Intercambios internacionales y estilos nacionales periféricos: aspectos de la mundialización de la ciencia. En Lafuente, A. et al. (Eds.) (1993), Mundialización de la ciencia y cultura nacional (pp. 725-733). Madrid: Doce Calles.

(1996). El proceso de institucionalización. En Salomón, J. et al. (Comps.), Una búsqueda Incierta. Ciencia, Tecnología y Desarrollo (pp. 199-233). México: Fondo Cultura Económica.

(2007). La movilidad científica desde la perspectiva de América Latina. En Vessuri, H., «O inventamos o erramos». La ciencia como idea-fuerza en América Latina. Bernal: Universidad Nacional de Quilmes (2014). Componentes culturales de la ciencia social en la era global. En Beigel, F. y Sabea, H. (2014), Dependencia académica y profesionalización en el Sur. Perspectivas desde la periferia (pp. 44-54). Mendoza: Ediunc; Rio de Janeiro: Sephis.

Shinn, T. (1999). Prólogo. En Krimer, P., De probetas, computadoras y ratones. La construcción de una mirada sociológica sobre la ciencia (pp. 13-24). Bernal: Universidad Nacional de Quilmes.

Libingstone, D. (2003). Putting science in its place. Geographies of scientific knowledge. Chicago: Chicago U. Press.

\section{Matharan, Gabriel}

«La dinámica centro-periferia en el estudio de la ciencia 
